\title{
Protease-Activatable Hybrid Nanoprobe for Tumor Imaging
}

\author{
Yaping Wang, Yifan Jiang, Meng Zhang, Jiao Tan, Jianming Liang, Huixin Wang, \\ Yaping Li, Huining He, Victor C. Yang,* and Yongzhuo Huang**
}

Tumor-associated proteases (TAPs), such as legumain, are actively involved in cancer progression; they have been used as biomarkers for diagnosis, prognosis, and drug targeting. As a result, in-vivo detection and trafficking of TAPs have attracted a great deal of attention. TAP-specific probes for in-vivo imaging, however, remain rare. A TAP-responsive hybrid nanoprobe system based on quantum dots (QD) and the fluorescence resonance energy transfer (FRET) effect is presented for the detection of legumain (asparaginyl endopeptidase), which is overexpressed in many tumors. A novel hybrid construction method is developed for fabricating the nanoprobe, by which the strong heparin-protamine affinity is used for conjugation. The hybrid comprises two components: 1) low-molecular-weight heparin (LH)-modified QD, and 2) low-molecular-weight protamine (LMWP)-conjugated fluorescence quencher QSY21, through a legumain-cleavable linker. The hybrid nanoprobe (i.e., a FRET system) is self-assembled via the LH-LMWP affinity. The linker between LMWP and QSY21 is selectively cleaved by legumain, leading to QSY21 detachment and fluorescence recovery in the tumor. In-vivo imaging is successfully achieved in the colon tumor mouse model. Importantly, such a hybrid nanoprobe system is adaptable for the detection of other TAPs (e.g., matrix metalloproteinase -2) by using an established, corresponding substrate-peptide linker, thereby offering a universal platform for TAP detection and tumor imaging.

\section{Introduction}

Proteases are involved in many biological and pathological processes via the regulatory mechanism of proteolysis, and consequently, they affect disease progression-typically in cancer, such as tumor angiogenesis, invasion, and metastasis. ${ }^{[1]}$

\footnotetext{
Y. Wang, Y. Jiang, M. Zhang, J. Tan, J. Liang, H. Wang, Prof. Y. Li, Prof. Y. Huang

Shanghai Institute of Materia Medica

Chinese Academy of Sciences

501 Haike Rd, Shanghai 201203, China

E-mail: yzhuang@simm.ac.cn

Y. Wang, Dr. H. He, Prof. V. C. Yang

Tianjin Key Laboratory on Technologies Enabling Development of

Clinical Therapeutics and Diagnostics

School of Pharmacy

Tianjin Medical University, Tianjin 300070, China

E-mail:vcyang@med.umich.edu

Prof. V. C. Yang

University of Michigan College of Pharmacy

428 Church St, Ann Arbor, MI 48109-1065, USA
}

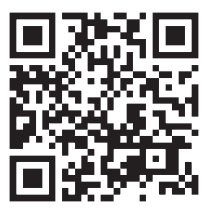

DOI: 10.1002/adfm.201400419
Tumor-associated proteases (TAPs) have become a central topic of interest in the scientific community because of their potential use as diagnostic and prognostic biomarkers and as drug targets. ${ }^{[2]}$ Realtime, in-vivo detection and monitoring of TAPs provide a powerful tool for the early diagnosis and screening of cancer. ${ }^{[3]}$ Moreover, a real-time TAP probe would also be helpful for improving our understanding of its in-vivo biological functions. Currently however, TAP-specific probes for invivo imaging remain rare.

Legumain (Enzyme Commission number: EC 3.4.22.34), an asparaginyl endopeptidase, is associated with malignant tumor invasion, proliferation, and metastasis, and it is highly expressed in the majority of solid tumors, including those of breast, colon, and prostate cancers. ${ }^{[4]}$ We also found that legumain is involved in a process from inflammation to the development of malignant colon tumors (data not shown). Legumain has been actively investigated for its clinical use as a biomarker for diagnosis, ${ }^{[5]}$ prognosis, ${ }^{[5]}$ and drug targeting. ${ }^{[6]}$ Legumain can selectively hydrolyze the asparaginyl carboxyl terminal. ${ }^{[7]}$ Such unique cleavage nature and its close association with cancer development and progression render legumain ideal as a target in the design of tumor-responsive prodrugs. ${ }^{[8]}$

Considering the importance of legumain, we present an activatable, legumain-responsive hybrid nanoprobe system for either in-vitro-cellular or in-vivo-tumor imaging based on the use of quantum dots (QDs) and the fluorescence resonance energy transfer (FRET) effect (Scheme 1). The hybrid nanoprobe system comprises two components: 1) QDs modified with low-molecular-weight heparin (LH), labeled as QD-LH; and 2) the fluorescence quencher "QSY21" conjugated to a peptide sequence labeled PEP, which is low-molecular-weight protamine (LP) and a legumain-cleavable peptide linker. We previously reported the application of heparin-LP as a reversible linker between two macromolecular components (e.g., the targeting antibody and enzyme drug) based on their charge interaction and affinity. ${ }^{[9]}$ Via the strong electrostatic affinity of LHLP, the fluorescent QD and quencher QSY21 can self-assemble into a hybrid nanoprobe, thereby forming a FRET system. Following intravenous (i.v.) administration, the nanoparticles will accumulate at the tumor target through the so-called "enhanced 


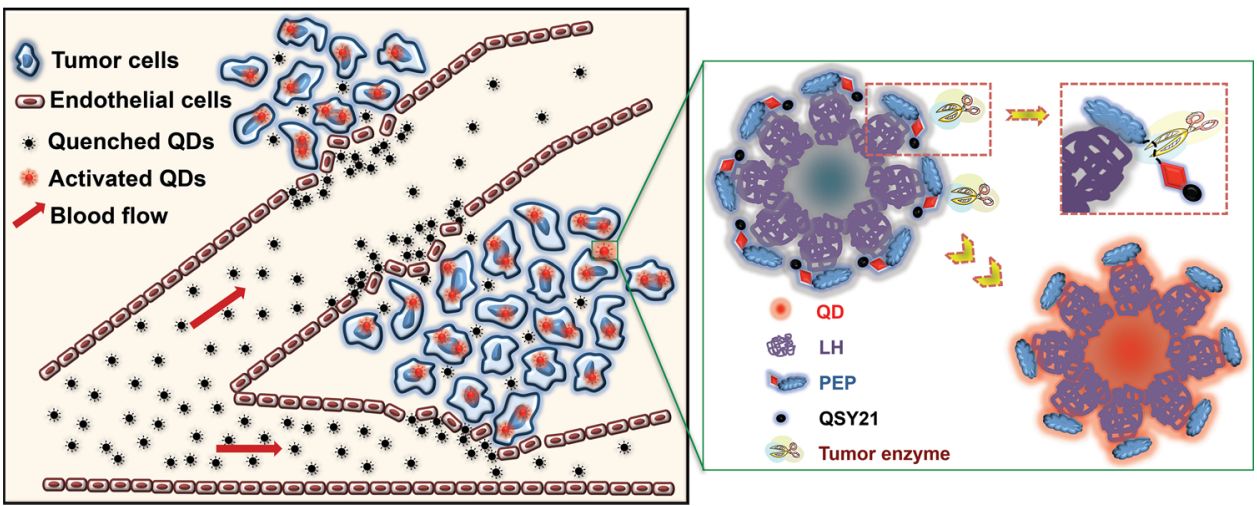

Scheme 1. Activatable nanoprobe comprises two components: 1) QD-LH: the fluorescence donor; and 2) the FRET quencher QSY21 coupled to a chimeric peptide (PEP) comprising LP and a legumain-cleavable sequence. The two components are linked via a tight but reversible electrostatic binding based on the LH-LP interaction, and thus form a FRET system (QD-LH/PEP-QSY), resulting in fluorescence quenching. The QSY21 quencher will detach once its linker is cleaved by legumain, and fluorescence is activated.

permeation and retention" (EPR) effect. ${ }^{[10]}$ The peptide linker will then be cleaved by legumain in the tumor, releasing the QSY21 quencher, and rendering the fluorescence of the recovered QD.

The proposed hybrid probe system offers several major benefits. First, conjugation of the donor and acceptor moieties at each end of a cleavable linker typically incurs extra effort and expense, due to the complicated synthesis route and the low yield. The automatic formation of the affinity-based LH-LP linkage in our hybrid system may be what is needed to ease such synthesis problems. Secondly, the hybrid QD system could serve as a generic platform technique for the development of imaging probes in the detection of various types of TAPs, simply by using a corresponding peptide linker that is specifically cleavable by the targeted protease to connect the QSY21 quencher to the LP, the LH binder. Last but not least, the nanoscale effect of the system allows for passive tumor targeting and increased tumor accumulation of the probes via the EPR effect.

In our studies, the hybrid nanoprobe was examined for its legumain-activatable imaging function under various conditions, including those of the cell culture, ex-vivo tumor slices, in-situ tissues, and in-vivo systemic targeted activation in the tumor.

\section{Results and Discussion}

The releasable QSY21 quencher was conjugated to the $\varepsilon$-amino group of the lysine of a strategically designed chimeric peptide (sequence: KPTNGGGVSRRRRRRGGRRRR; K: lysine, P: proline, T: threonine, N: asparagine, G: glycine, V: valine, S: serine, R: arginine) comprising a legumain-cleavable substrate and LP; the substrate sequence of PTN can be recognized and specifically cleaved by legumain. This conjugate was termed PEP-QSY. Triglycine (GGG), used as a flexible peptide tether, was incorporated between the legumain-recognizable substrate (KPTN) and the LP (VSRRRRRRGGRRRR) in order to provide increased accessibility to the legumain-cleavable substrate, thereby enhancing the cleavage efficiency. Via the electrostatic affinity between LH and LP, the activatable QD-based FRET nanoprobe system (QD-LH/PEP-QSY, further abbreviated to $\mathrm{QD} / \mathrm{QSY}$ ) was formed. Fluorescence of the $\mathrm{QD}$ was significantly inhibited by increasing the amount of the attached quencher of PEP-QSY, and the intensity was reduced to around $10 \%$ when the QD/QSY ratio reached 3:1, but with no further reduction in intensity beyond this ratio (Figure $1 \mathrm{~A}$ ). Hence, the ratio of 3:1 was selected for the subsequent investigations. The nanoprobe of QD/QSY was characterized using dynamic light scattering (DLS) and transmission electron microscopy (TEM) (Figure 1B,C). As shown in Figure 1D, the fluorescence of the nanoprobe remained stable for up to $120 \mathrm{~h}$ in both phosphate buffered saline (PBS) and cell culture medium (DMEM, Dulbecco's Modified Eagle's Medium) at $37^{\circ} \mathrm{C}$. The QD-LH and its FRET-based nanoprobe displayed remarkable biocompatibility with cells when using the model non-tumoral 293T cell line; nearly $80 \%$ cell viability was observed even at a nanoparticle concentration as high as $1.5 \mathrm{mg} / \mathrm{mL}$ in (Figure 1E). The findings suggest the safety and potential of the nanoprobe for use in in-vivo studies.

A preliminary activation investigation was conducted by adding protamine to the QD/QSY system. As previously demonstrated by our study of the strategy termed ATTEMPTS (antibody-targeted, triggerable, electrically modified prodrug-type strategy), ${ }^{[11]}$ protamine has a stronger affinity to LH than to LP and can thus cause the detachment of PEP-QSY component from the QD/QSY complex. Over $80 \%$ recovery of the fluorescence was achieved after the addition of an adequate amount of protamine to the QD/QSY hybrid nanoprobe (Figure 2A,B), indicating the successful reversal of the deactivated FRET system. Subsequent to this study, enzymatic activation of the nanoprobe by legumain was examined. Fluorescence of the nanoprobe was efficiently activated following by the addition of legumain (Figure $2 \mathrm{C}, \mathrm{D}$ ). In contrast, almost no recovery of the fluorescence was obtained when a non-specific enzyme trypsin was used, due to its inability to cleave away the quencher component (PEP-QSY) from the QD/QSY (Figure 2E,F), indicating the specificity of activation of this system by legumain.

Cellular activation of the QD/QSY system was performed using both the normal HEK 293T cell line and the 

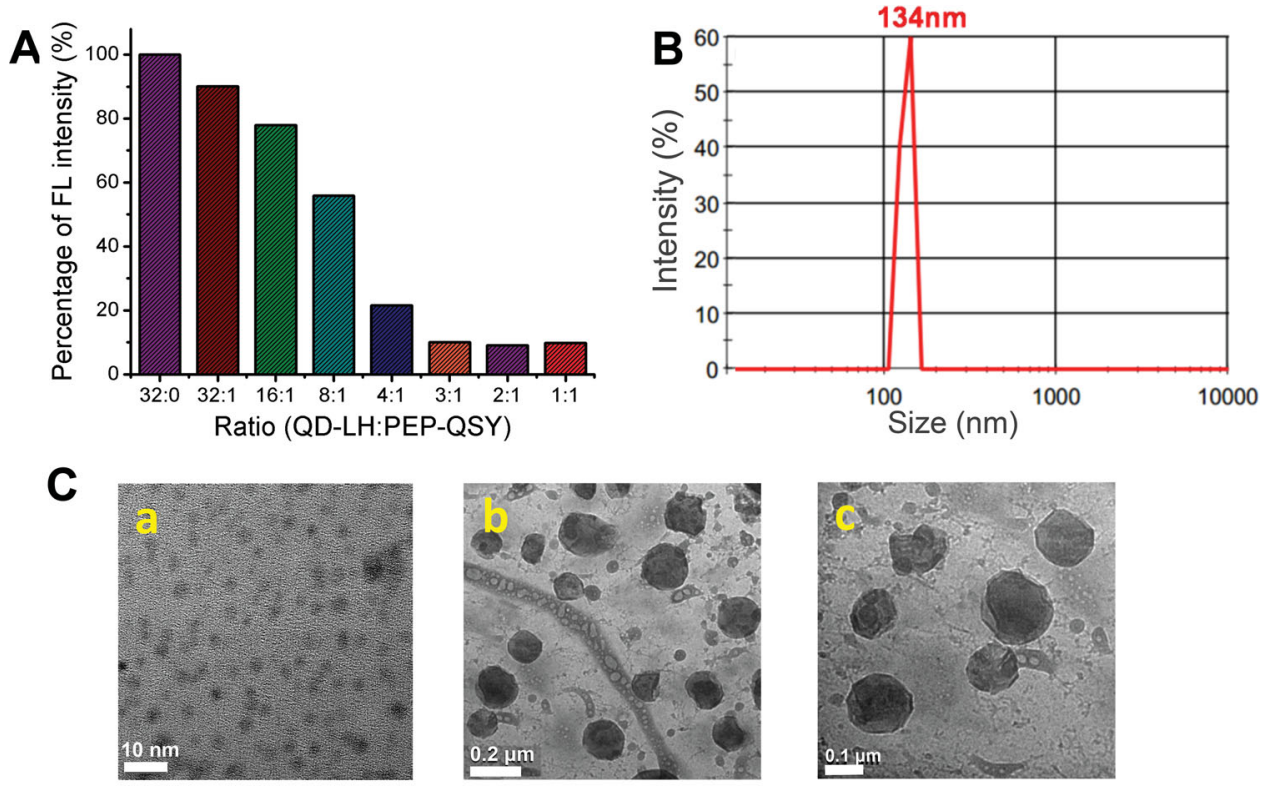

\section{D}

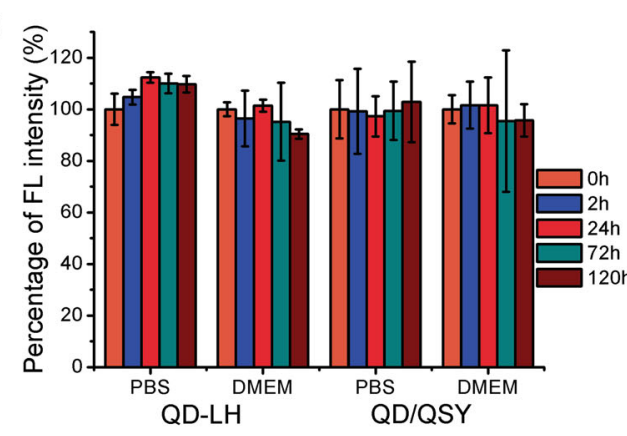

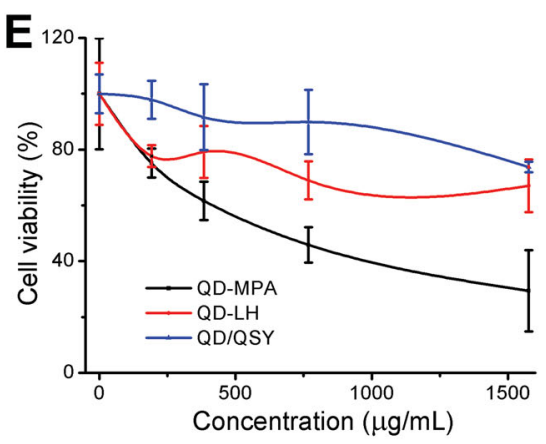

Figure 1. Characterization of the QD/QSY system (QD-LH/PEP-QSY). A) Enhanced fluorescence quenching efficiency related to the increase of the QD/QSY ratios. B) Particle size of QD/QSY nanoprobe determined by DSL. C) TEM images of a) LH-modified QDs and b,c) the QD/QSY nanoprobe. D) QD-LH and the nanoprobe remained stable in PBS and DMEM cultured medium, as indicated by the fluorescence. E) Cell-viability studies showing that QD-LH and the QD/QSY nanoprobe were low in cytotoxicity; QD-MPA are the QDs coated with 3-mercaptopropionic acid (MPA).

legumain-transfected HEK 293T cells (293T-Leg). Whereas the fluorescence of the nanoprobe remained quenched in the normal HEK 293T cells, it was nevertheless significantly activated in the 293T-Leg cells (Figure 3), suggesting the practicality of the nanoprobe in imaging legumain-positive cancer cells. Whereas the fluorescence of the nanoprobe remained quenched in the normal HEK 293T cells, it was nevertheless significantly activated in the 293T-Leg cells (Figure 3), suggesting the practicality of the nanoprobe in imaging legumain-positive cancer cells.

The cellular activation efficiency of the nanoprobe was also examined in cellular cultures using a fluorescence-activated cell sorter (FACS). The fluorescence-positive percentage in the 293T-Leg cells treated with varying concentrations of the probe was $>90 \%$, while that in the normal 293T cells was $25-39 \%$ (Figure 4A,B). The median fluorescence intensity in the 293TLeg cells was 2-3 times higher than that in normal 293T cells (Figure 4C). The FACS results demonstrate the legumaininduced activation of the prodrug-type probe. The HEK 293T cells also had very minor legumain expression, as shown in Figure 4D. Therefore, it caused slight activation. Moreover, the serum proteases may result in low non-specific activation. However, the increase in activation efficiency in the HEK 293TLeg cells are very significant compared to that in HEK 293T cells (e.g., $93 \%$ versus $25 \%$ at a nanoprobe concentration of $80 \mu \mathrm{g} / \mathrm{mL})$.

The activation process within the cell was further examined by using confocal laser scanning microscopy (CLSM). The initial fluorescence activation of the nanoprobe apparently occurred on the membrane or in the extracellular space (Figure 5A,B); it was then transported into the cell (Figure 5C,D). Interestingly, at the end of the study, the QD fluorescence was transferred back to the extracellular space again, probably via the cellular event of exocytosis. Our previous observations indicated that a QD could be exocytosed with a half-life of 21 min. ${ }^{[12]}$ Formation of the active legumain is a $\mathrm{pH}$-dependent multi-step process in the endosome/lysosome system. ${ }^{[13]}$ The mature legumain present in the cell membrane and released into the extracellular space is believed to control extracellular matrix remodeling. ${ }^{[14]}$ Our observations provided additional evidence to support the extracellular enzymatic activity monitored by the nanoprobe. 

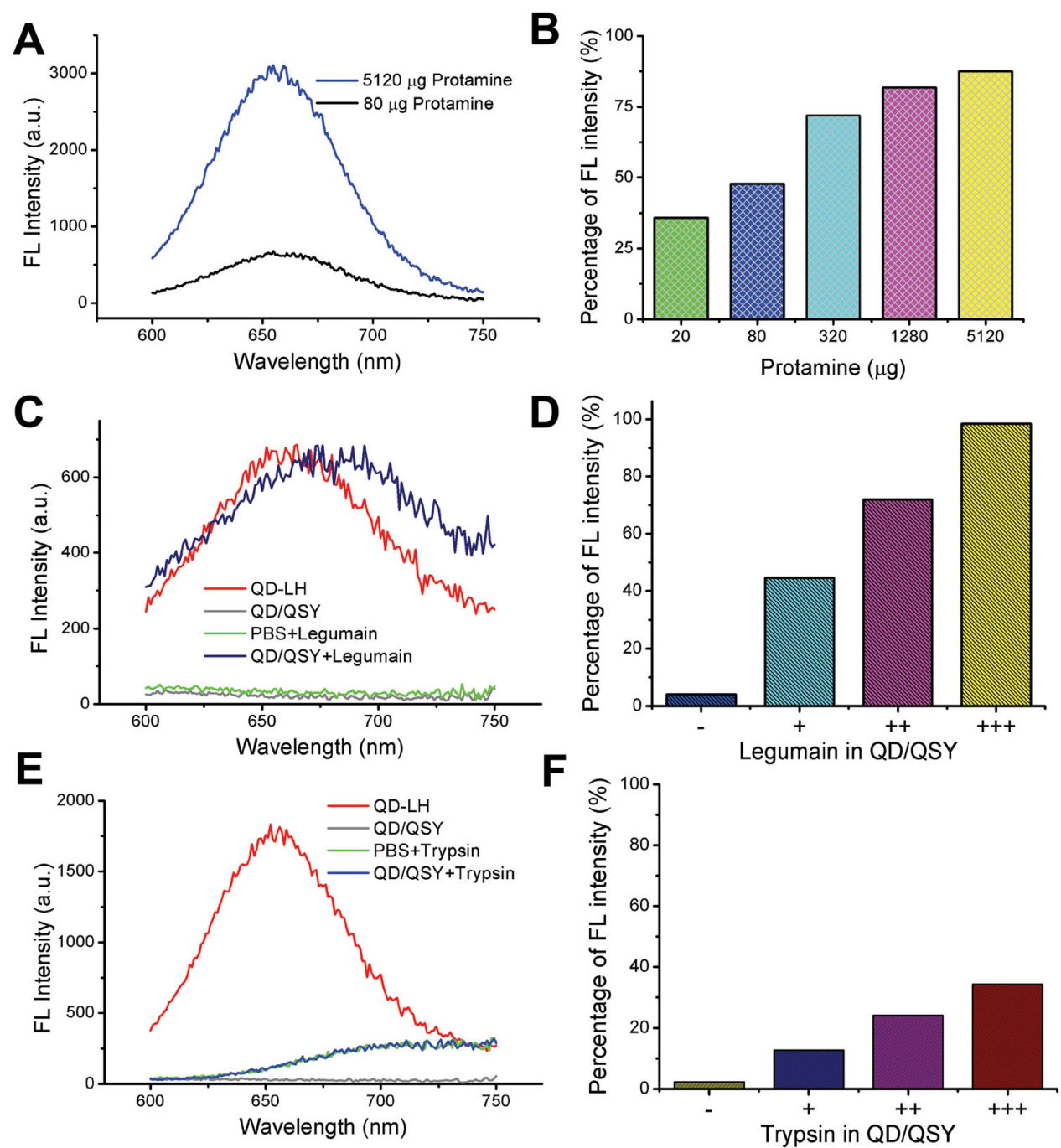

Figure 2. Activation of the QD/QSY nanoprobe (QD-LH/PEP-QSY). A) Fluorescence recovery can be triggered by adding enough protamine to substitute the PEP-QSY of the QD/QSY nanoprobe. B) Enhanced fluorescence recovery by increasing the amount of protamine. C) Fluorescence recovery triggered by legumain. D) Enhanced fluorescence recovery by increasing the concentration of legumain. E) Only minor fluorescence recovery was triggered by the non-specific enzyme trypsin. F) No significant recovery of fluorescence intensity even when a high concentration of trypsin was employed.

The tissue-specific activation of the prodrug-type probe was examined ex vivo using tissue cryosectioning slices that included the tumor and major organs collected from the mice harboring the human colon SW620 xenograft tumor with legumain overexpression. The probe concentration was $160 \mu \mathrm{g} / \mathrm{mL}$. As expected, activation of the quenched nanoprobe thereby yielding fluorescence occurred within these tumor slices (Figure 6). In contrast, fluorescence was not observed in the glutaraldehyde-pretreated tumor slices, because of the denaturation of legumain by glutaraldehyde. This further confirmed the enzyme-related activation process. Moreover, fluorescence activation was not found in slices of major organs, apparently due to the lack of legumain expression in these tissues. These ex-vivo findings were in good agreement with the in-vitro results, yielding strong support for the selective and specific imaging of legumain-presenting tumor tissues by the hybrid nanoprobe.
To examine the nanoprobe system in situ, mice bearing legumain-overexpressed colon SW620 xenograft tumor were used to study the activation event. As displayed in Figure 7A and $\mathrm{B}$, the fluorescence intensity peaked $11 \mathrm{~min}$ after peritumoral injection of the probe, with the ROI (region of interest) value of $2.267 \times 10^{6}$, clearly defining the target from its surroundings. However, the back (or spine) also displayed fluorescence signals after $11 \mathrm{~min}$. Because it is at the higher position and is closer to the light source and charge-coupled device (CCD) camera compared to other organs or tissues, it usually causes the increased refraction of light and the strengthened signals, particularly when using the fluorescence probe with emission wavelength less than $700 \mathrm{~nm}$. Moreover, in order to evaluate the effect of non-specific in-vivo activation, the probe was administered via intramuscular injection through the left thigh to the same animal at the 24-min mark after the animal receiving peritumoral administration. As shown in Figure 7C, 


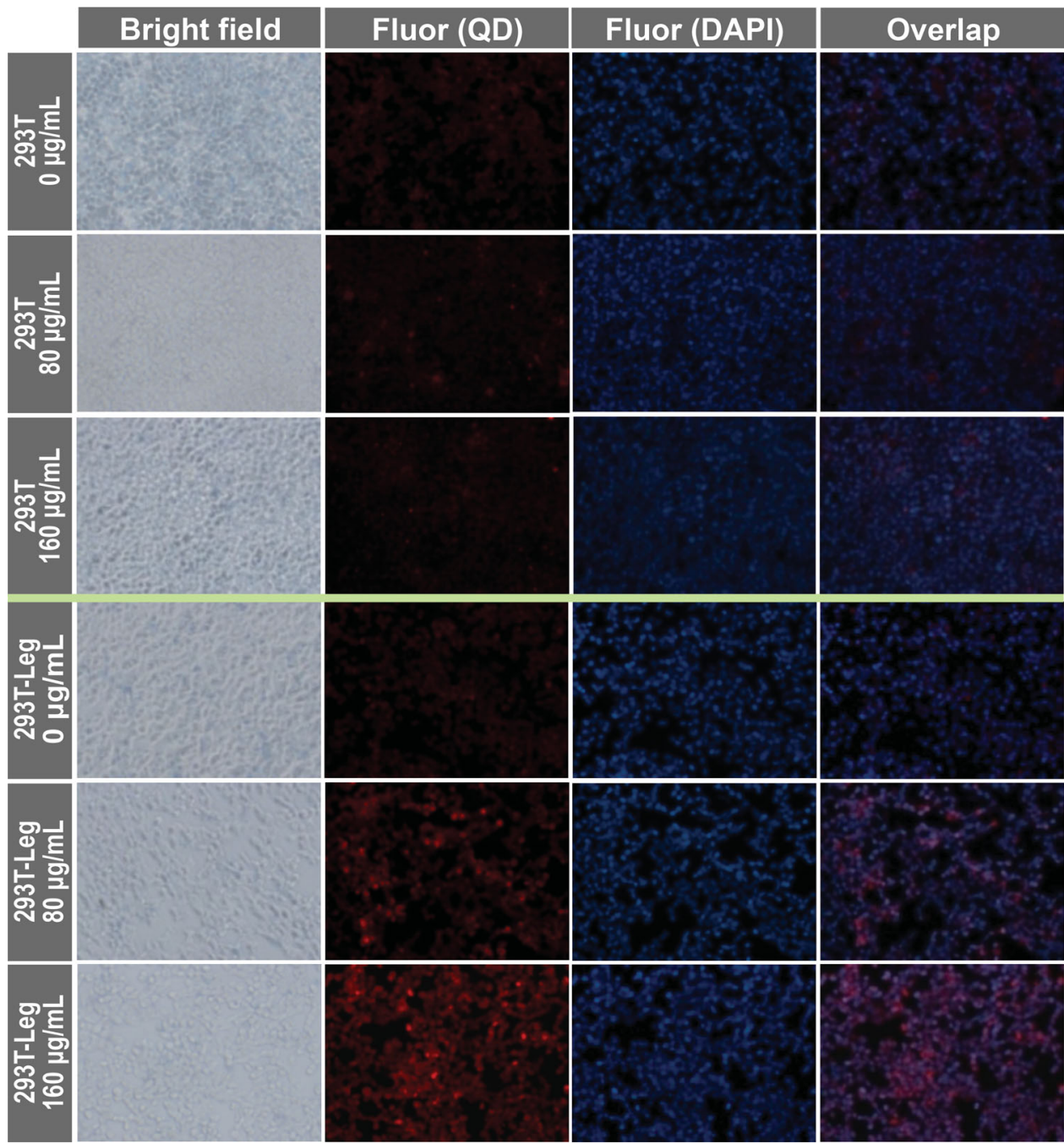

Figure 3. Activation of the QD/QSY nanoprobe at varying concentrations $(0,80,160 \mu \mathrm{g} / \mathrm{mL})$ in the HEK $293 \mathrm{~T}$ cell line and the legumain-transfected HEK 293T cells (293T-Leg). Images presented are bright-field, red fluorescence (Fluor (QD)), blue fluorescence (DAPI = 4',6-diamidino-2-phenylindole) and merged (Overlap) images. The nanoprobe was efficiently activated in the 293T-Leg cells, whereas it largely remained quenched in the normal 293T cells.

merely a slight activation was detected, estimated from the peak fluorescence intensity at the muscle injection site. The results further confirmed that activation of the FRET-based nanoprobe could primarily be activated specifically at the tumor site, due to the presence of the overexpressed legumain.

To examine the practical feasibility and applicability of the nanoprobe for in-vivo imaging and legumain-detection, quenched nanoprobe was administered through the conventional tail-vein route. Under such conditions, the preferential tumor accumulation of the nanoprobe could be obtained by taking the advantage of the tumor-associated EPR effect. As revealed in Figure 8A, the fluorescence intensity in the tumor significantly increased with time-from 0.5 to $2 \mathrm{~h}$; it then plateaued until the 24-h mark, clearly distinguishing the tumor from other tissues. Normalized fluorescence intensity showed that the fluorescence signal in the tumor $24 \mathrm{~h}$ after i.v. injection was 1.62 times stronger than that at $0.5 \mathrm{~h}$ (Figure 8B). The major organs were collected $24 \mathrm{~h}$ after i.v. injection to better monitor tissue-specific activation of the nanoprobe. The normalized fluorescence intensity showed that the signal in the ex-vivo tumor was 6.0, 2.3, 4.7, 3.4, 5.8, and 4.9 times stronger than that in the heart, liver, spleen, kidney, and brain, respectively (Figure 8C,D). It should be pointed out that the fluorescence distribution in the normal organs was mainly caused by the metabolic degradation of the peptide-based quencher and non-specific cleavage of the linker, thereby contributing to mild non-specific activation. Taken together, these results further demonstrate the potential and in-vivo applicability of the QD/QSY nanoprobe in the selective detection of legumainpresenting tumors by responding to the legumain-rich tumor microenvironments.

A primary benefit of this QD/QSY nanoprobe system lies in the universal applicability of the established hybrid construction method. This method possesses great versatility and utility 

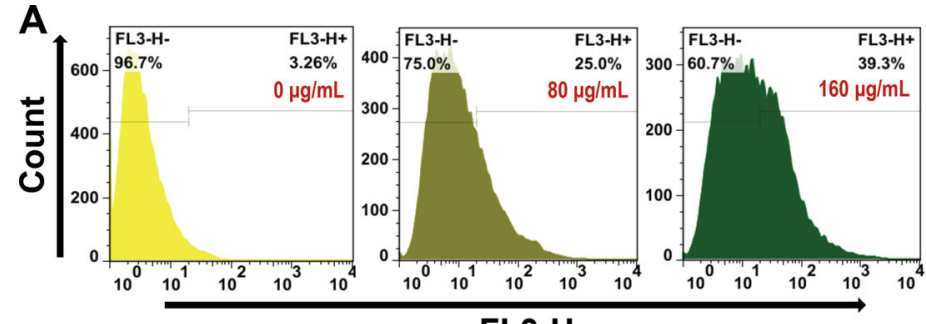

\section{C}
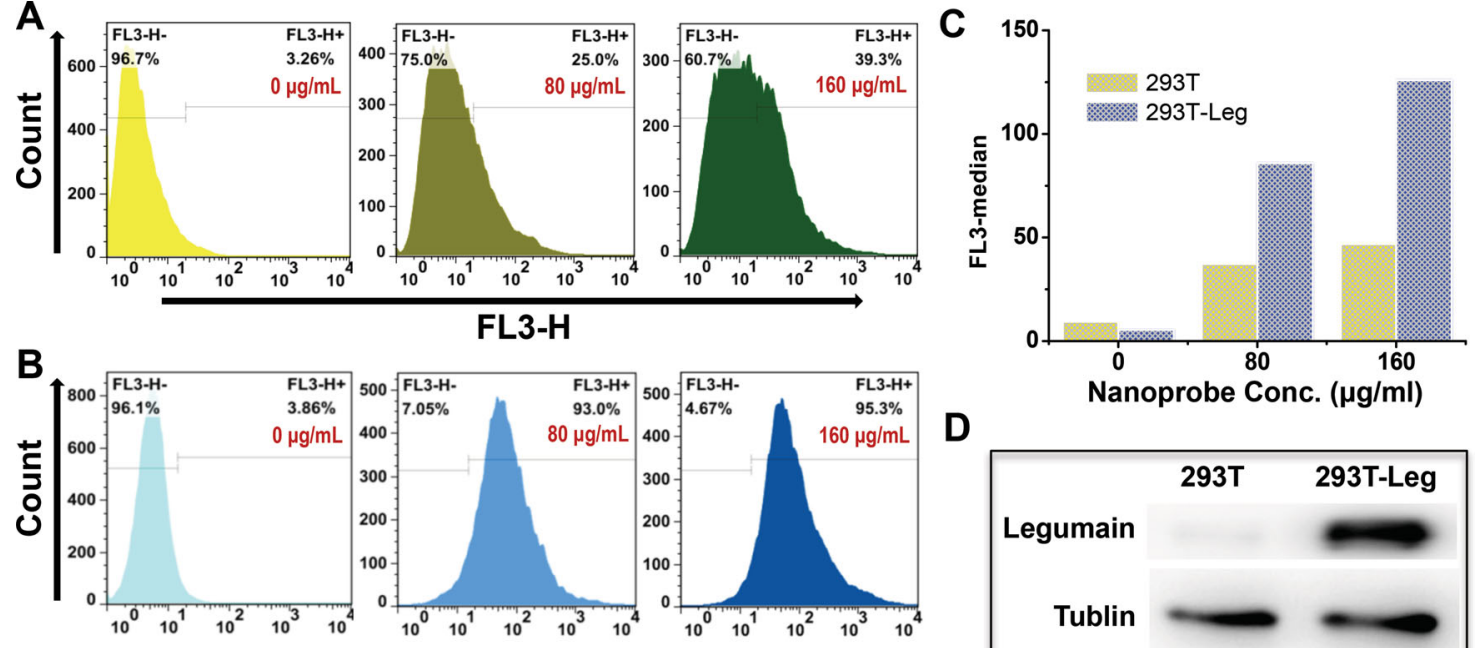

D

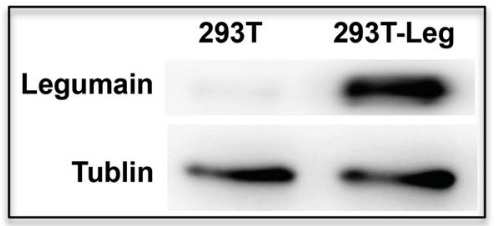

Figure 4. FACS assay for legumain-induced activation of the QD/QSY nanoprobe. Various nanoprobe concentrations $(0,80$, and $160 \mu \mathrm{gg} / \mathrm{mL}) \mathrm{were}$ employed in the investigation. A) FACS results for normal 293T cell line, showing minor activation. B) FACS results for the legumain-transfected 293T cells (293-Leg), showing increased activation relative to the normal cell line (FL3-H: height of red fluorescence intensity). C) The median fluorescence intensity observed in the normal 293T cells and transfected 293T-Leg cells. D) Western blotting analysis, showing very minor legumain expression in 293T cells but overexpression in 293-Leg cells.

for fabricating a variety of activatable QD systems targeting different TAPs. Indeed, aside from legumain, we also demonstrated the feasibility for in-vivo detection of other TAPs, such as matrix metalloproteinase 2 (MMP-2), simply by substituting the original legumain-cleavable peptide with a substrate cleavable by MMP-2 in the activatable QD system (Figure 9). With this in mind, the presented adaptable strategy could lead to the development of a new paradigm of specially tailored QD-based imaging platforms for tumors carrying specific, overexpressed protease biomarkers.

The general mechanism for protease-activatable probe design is a FRET effect that is achieved by employing a substrate-peptide as a cleavable linker between the FRET fluorophore and

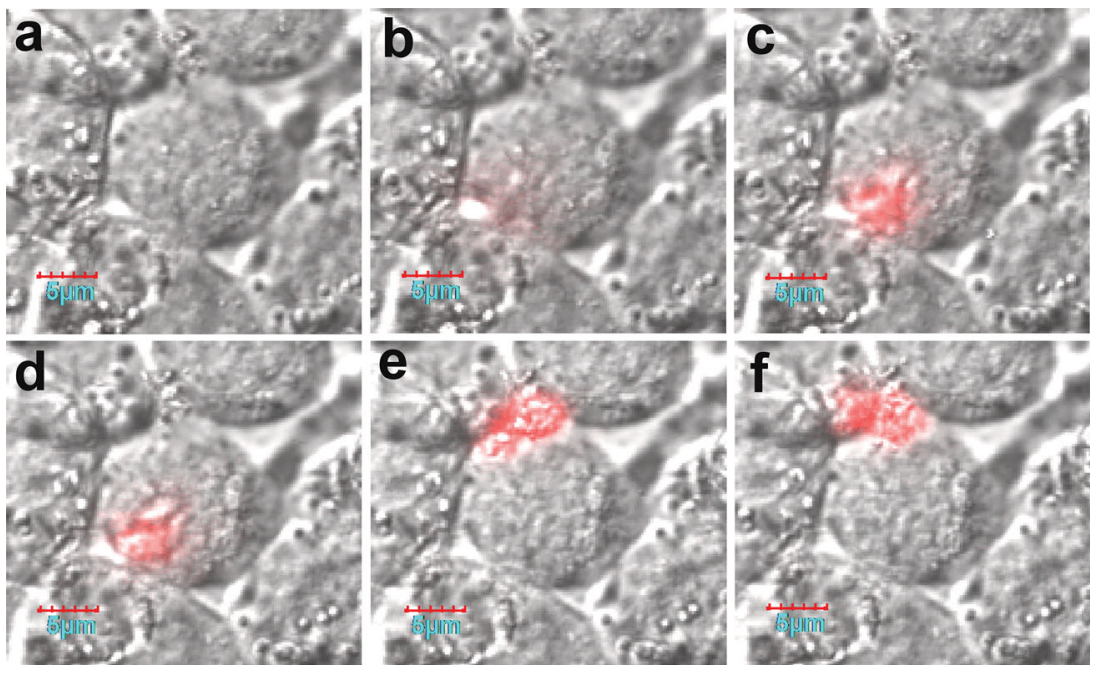

Figure 5. The activation process as monitored by CLSM: images at a) 49 , b) 62 , c) 64 , d) 74 , e) 81 , and f) $86 \mathrm{~min}$. quencher. To enhance peptide stability and reduce non-specific cleavage in vivo, the costly D-forms of peptide sequences are necessary to ensure image quality, especially in those cases involving long peptides (e.g., hairpin structure). ${ }^{[15]}$ However, although natural amino acids are used in our system, non-specific activation was insignificant, indicating the additional benefits of our strategy.

\section{Conclusion}

In summary, we report the development of a novel, proteaseresponsive QD nanoprobe platform for detecting tumor-associated protease biomarkers and for tumor imaging. Molecular imaging of legumain was successfully achieved under cellular, exvivo tissue-slice, in-situ, and in-vivo conditions, providing a promising tool for cancer detection. Importantly, based on the adaptability of this hybrid nanoprobe construction technology, it is envisioned that this imaging system could be applied universally to monitor tumors with known specifically overexpressed TAPs, simply by using the corresponding protease-substrate peptide that serves as a linker between LMWP and the fluorescence quencher. Unlike the conventional probes that typically need to be custom-made for each targeted, specific protease, the LHmodified QD serves as a universal fluorescent moiety. The LH-LMWP-based affinity linkage provides great convenience for the hybrid construction, easing the fabrication process. This is a versatile method for the 


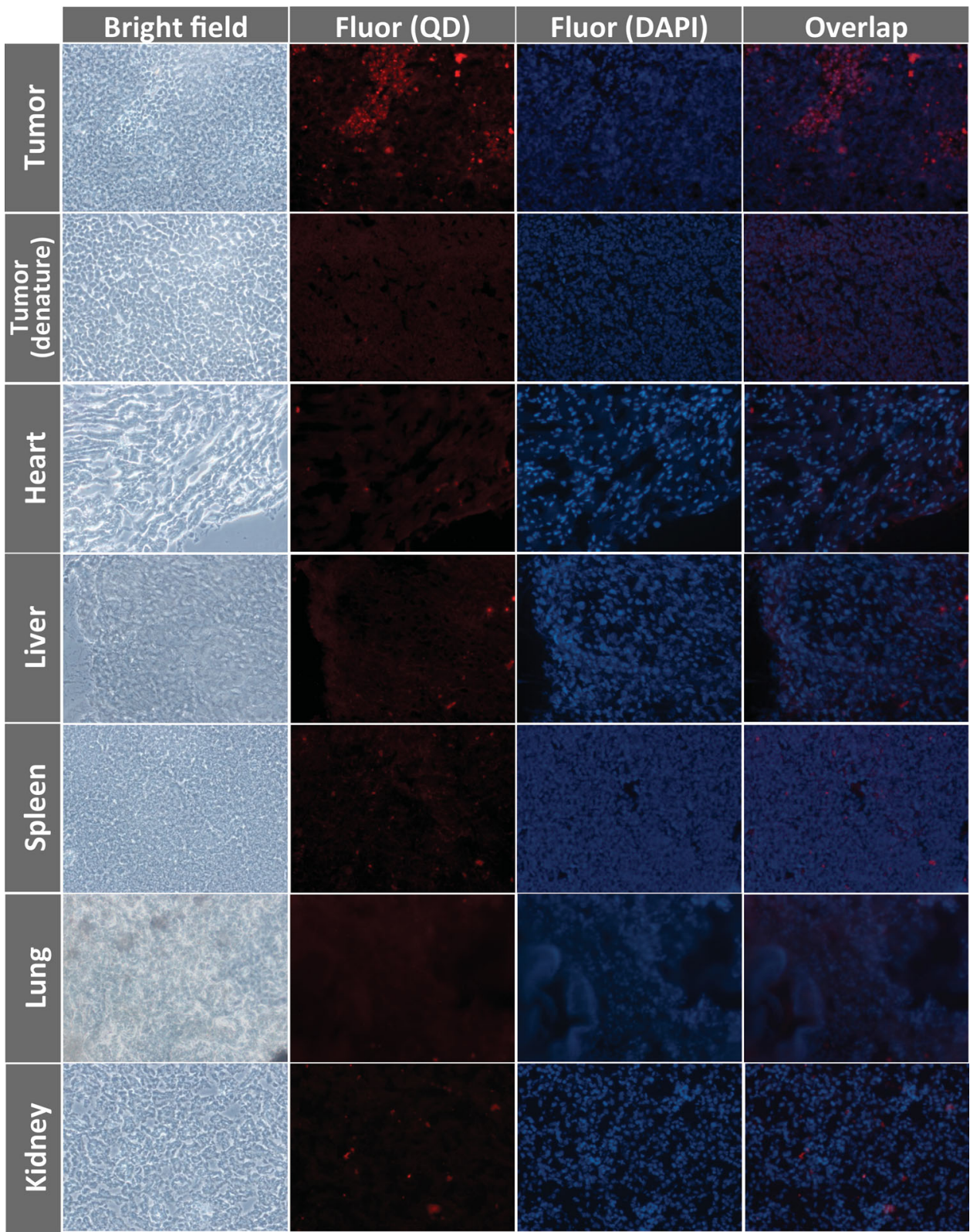

Figure 6. Ex-vivo detection of the tumor-specific nanoprobe activation. Images presented are bright-field, red fluorescence (QD), blue fluorescence (DAPI), and merged (Overlap) images. The FRET-based nanoprobe was activated in legumain-presenting tumor tissue (cryosection slide), but it was not activated in the legumain-denatured tumor that was pretreated with glutaraldehyde nor in other normal tissues that did not express legumain.

fabrication of a protease-activatable probe for in-vivo detection and tumor imaging.

\section{Experimental Section}

Materials: $\mathrm{NaBH}_{4}$, Tellurium powder, $\mathrm{CdCl}_{2}, \mathrm{NalO}_{4}$, and cysteamine hydrochloride were obtained from Sinopharm Chemical Reagent Co., Ltd. (Shanghai, China). MPA was obtained from Alfa Aesar (UK). LH (Enoxaparin Sodium) was obtained from Changshan Biochemical Pharmaceutical Co., Ltd (Shijiazhuang, China). GelRed was purchased from Biotium (Hayward, USA). Fetal bovine serum (FBS), DMEM, penicillin-streptomycin, $0.25 \%$ trypsin-EDTA solution
(EDTA = ethylenediaminetetra-acetic acid), dimethyl sulfoxide (DMSO), QSY21-NHS (where NHS is N-hydroxysuccinimide), and 3-(4,5-dimethylthiazol-2-yl)-2,5-diphenyltetrazolium bromide (MTT) were obtained from Invitrogen (USA). The Micro BCA protein assay kit and trypan blue were acquired from the Beyotime Institute of Biotechnology (Haimen, China). All other reagents were of analytical grade.

Preparation of the Prodrug-Type Probe: 1) MPA-Coated CdTe Quantum Dots (QD-MPA): Tellurium powder $(0.2 \mathrm{mmol})$ and $\mathrm{NaBH}_{4}(1.3 \mathrm{mmol})$ were heated at $80{ }^{\circ} \mathrm{C}$ for $1 \mathrm{~h}$ under the protection of $\mathrm{N}_{2}$ in order to prepare the $\mathrm{NaHTe}$ solution. $\mathrm{A} \mathrm{CdCl}_{2}$ solution $(0.2 \mathrm{mmol})$ and a MPA solution $(0.34 \mathrm{mmol})$ were mixed togetherb and the $\mathrm{pH}$ was adjusted to 12 . The $\mathrm{NaHTe}$ solution $(0.02 \mathrm{mmol})$ was added to the $\mathrm{Cd}$ precursor solution at a reaction ratio of 1:1.7:0.1 (Cd:MPA:Te). The reaction 


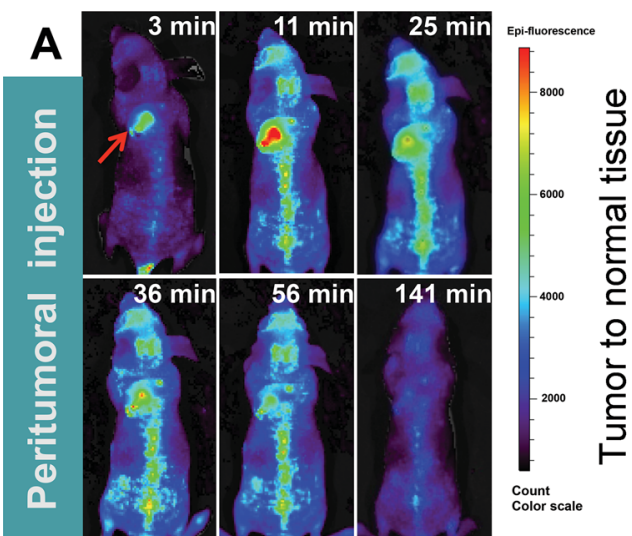

B
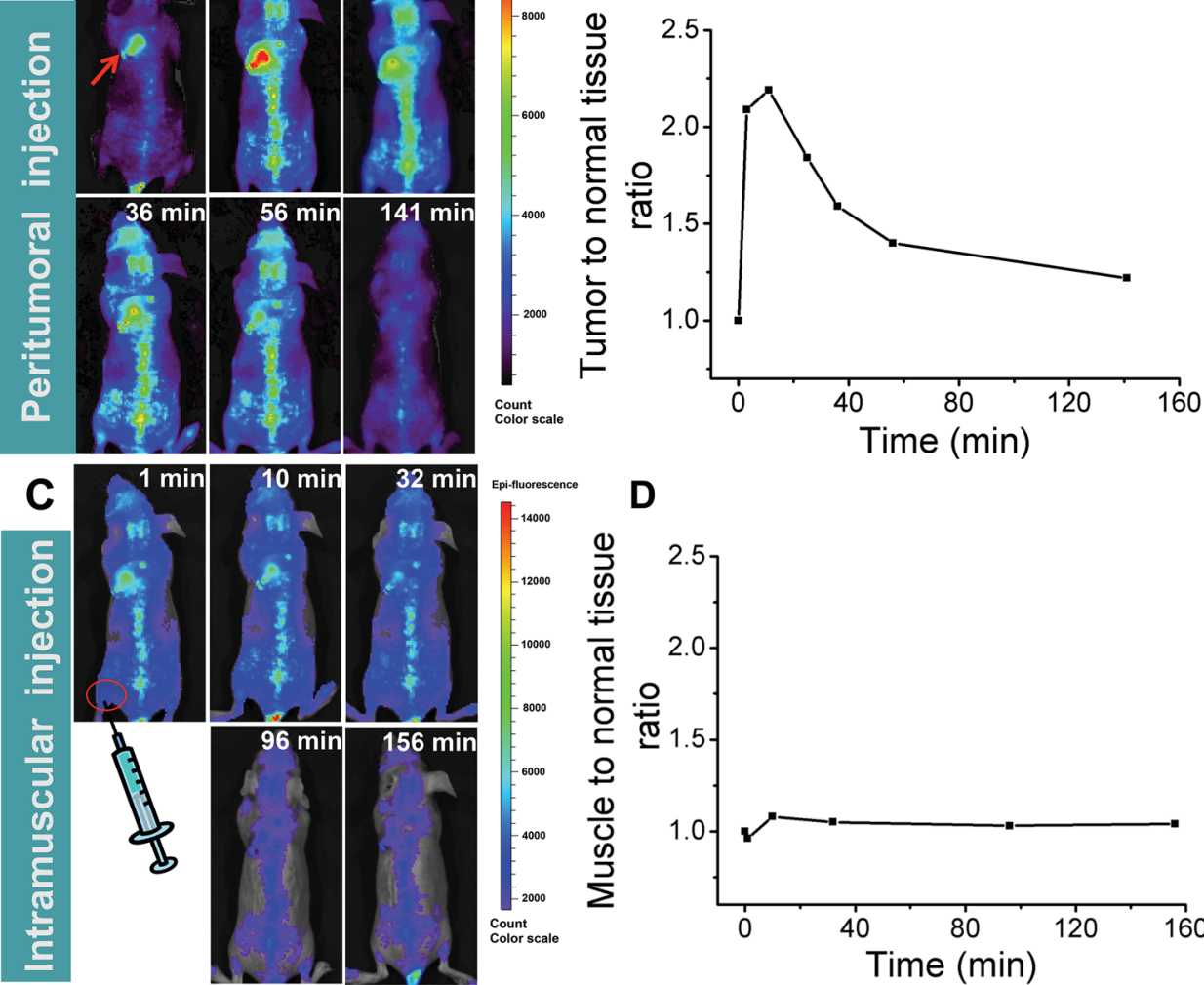

D

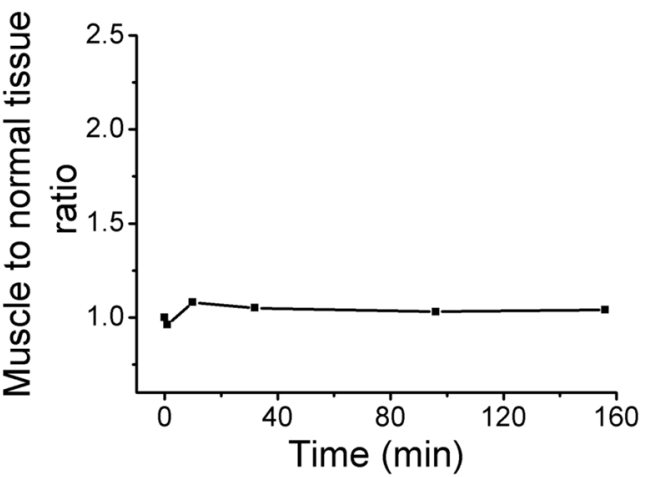

Figure 7. In-vivo imaging following peritumoral and intramuscular injections. A) Fluorescence omages taken at various time points from 3 to 141 min following peritumoral injection of the nanoprobe. B) Plots for the ratios of the fluorescence intensity in the tumor versus in normal tissues following peritumoral injection. C) Fluorescence Images taken at various time points from 1 to 156 min following intramuscular injection of the nanoprobe. D) Plots for the ratios of the fluorescence intensity in the tumor versus in normal tissues following intramuscular injection.

mixture was then heated to reflux under $\mathrm{N}_{2}$ flow. The thus-formed CdTe QDs were purified by precipitation using isopropanol, followed by vacuum drying.

Preparation of the Prodrug-Type Probe: 2) Thiol LH (LH-SH): LH $(50 \mathrm{mg}$ ) was oxidized by sodium periodate in $10 \mathrm{~mL}$ of citric acid buffer $(0.1 \mathrm{M}, \mathrm{pH} 7.0)$ at a molar ratio of 1:1.5 ( $\mathrm{NaIO}_{4}$ : ( $\mathrm{LH}$ repeating unit)). After purification by dialysis, the product was reacted with cysteamine hydrochloride $(11 \mathrm{mg})$ in $20 \mathrm{~mL}$ of phosphate buffer $(0.1 \mathrm{~m}, \mathrm{pH} 7.0)$. Thiol LH was obtained after reduction using sodium borohydride $(125 \mathrm{mg})$ and purification by dialysis.

Preparation of the Prodrug-Type Probe: 3) Ligand-Exchange Reaction from QD-MPA to QD-LH: The LH-SH was added to the QD-MPA solution in PBS ( $\mathrm{pH} 7.4)$, and incubated overnight at room temperature. The resultant QD-LH was purified by precipitation using isopropanol, followed by vacuum drying.

Preparation of the Prodrug-Type Probe: 4) Coupling of QSY21 to PEP: The chimeric peptide of LP and the legumain-cleavable substrate was synthesized via a solid-state method (sequence: KPTNGGGVSRRRRRRGGRRRR, with N-terminal acetylated and C-terminal amidated, as denoted PEP). PEP in DMSO $(10 \mu \mathrm{g} / \mathrm{mL})$, via the $\varepsilon$-amino group on the terminal lysine, was reacted with the QSY21-NHS ester $(1.3: 1 \mathrm{~mol} / \mathrm{mol})$ in the presence of $1 \%$ $\mathrm{N}$-methylmorpholine for $48 \mathrm{~h}$. The product of QSY21-PEP (or PEP-QSY) was determined and purified using reverse-phase $\mathrm{C} 18$ high-performance liquid chromatography (HPLC). For quantitative analysis of PEP-QSY, the HPLC conditions were: analytic HPLC system (Agilent-1200, USA) with a C18 column $(4.6 \mathrm{~mm} \times 250 \mathrm{~mm}, 5 \mu \mathrm{m}) ; \lambda=210 \mathrm{~nm}$; flow rate of $1 \mathrm{~mL} /$ min; gradient elution using $\mathrm{H}_{2} \mathrm{O} /(0.1 \%$ trifluoroacetic acid (TFA)) and $\mathrm{CH}_{3} \mathrm{CN} /(0.1 \%$ TFA) as solvent $\mathrm{A}$ and $\mathrm{B}$, and running from $(95 \%$ $\mathrm{A} / 5 \% \mathrm{~B})$ to $(30 \% \mathrm{~A} / 70 \% \mathrm{~B})$ in 30 min. The purification of PEP-QSY was performed by a preparative HPLC (Waters 1525 system, USA) with a C18 column $(10 \mathrm{~mm} \times 150 \mathrm{~mm}, 5 \mu \mathrm{m})$. Chromatographic conditions were as follows: $\lambda=280 \mathrm{~nm}$; flow rate of $5 \mathrm{~mL} / \mathrm{min}$; gradient elution using $\mathrm{H}_{2} \mathrm{O} /(0.1 \%$ TFA $)$ and $\mathrm{CH}_{3} \mathrm{CN} /(0.1 \%$ TFA $)$ as solvent $\mathrm{A}$ and $\mathrm{B}$, respectively, and running from $(95 \% \mathrm{~A} / 5 \% \mathrm{~B})$ to $(25 \% \mathrm{~A} / 75 \% \mathrm{~B})$ in $10 \mathrm{~min}$.

Preparation of the Prodrug-Type Probe: 5) Self-Assembly of PEP-QSY with QD-LH (QD/QSY): The self-assembly of PEP-QSY with QD-LH nanoparticles was prepared by mixing PEP-QSY, in a range of 0-32 $\mu \mathrm{g}$, with QD-LH $(32 \mu \mathrm{g})$ in deionized water. The mixtures were incubated for $30 \mathrm{~min}$ at room temperature. The FRET efficiency related to the addition of the quencher (PEP-QSY) was monitored by measuring the fluorescence intensity using a fluorospectrometer (Hitachi F4600, Japan).

Characterization of the Prodrug-Type Probe: The sulfhydryl content of the LH-SH was measured using Ellman's reagent, according to the manufacturer's protocol (Thermo Scientific Piercer, USA).

Size analysis and the $\xi$ potential of QD-LH were performed using a Zetasizer Nano-ZS90 (Malvern Instrument). The morphology was observed using TEM at an accelerating voltage of $100 \mathrm{kV}$ (JEOL JEM-1200EX, Japan). Fluorescence intensity was measured on a fluorospectrometer (Hitachi, F4600, Japan).

Preliminary activation of the prodrug-type probe was carried out by adding protamine to competitively substitute the PEP-QSY and thus release QD-LH from the original self-assembly; fluorescence intensity was measured using the fluorospectrometer. 

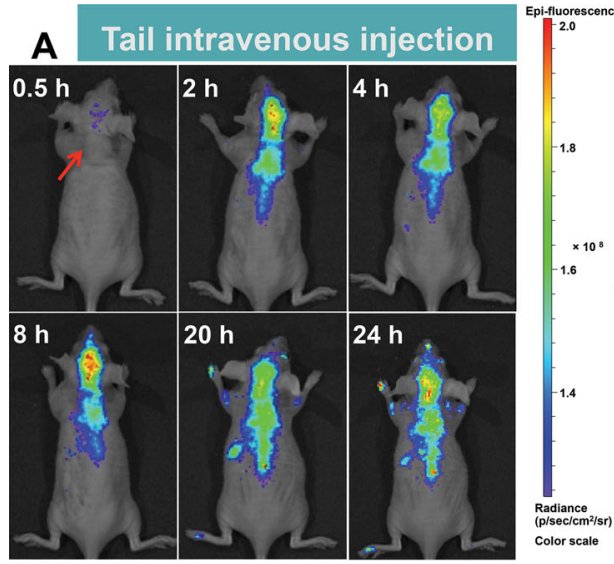

\section{B}
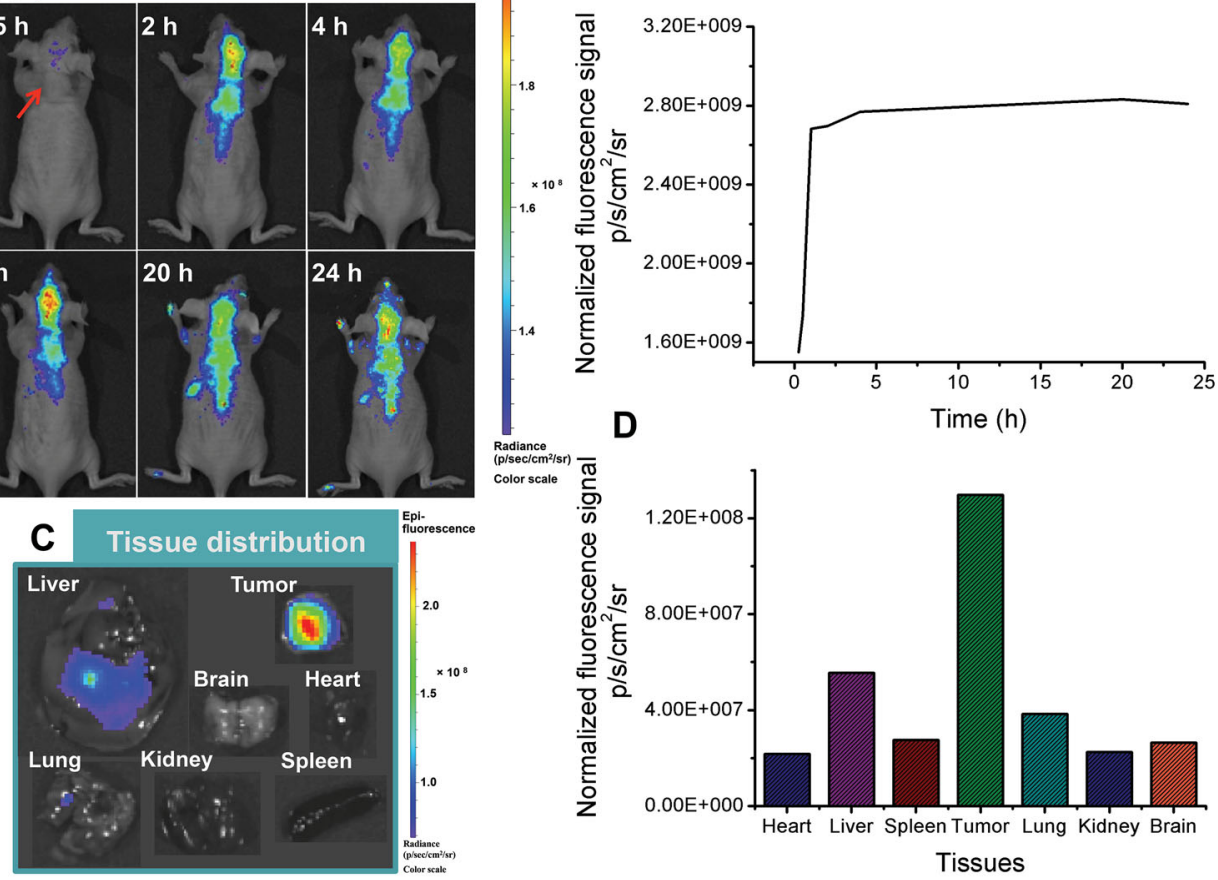

Tissues

Figure 8. In-vivo imaging following tail i.v. injection. A) Fluorescence images taken at various time points after injection: 0.5 to 24 h. B) Normalized ratios of the fluorescence intensity in tumor versus other major tissues. C) Ex-vivo tissue imaging at the experimental endpoint. D) The fluorescence intensity in the collected tissues represented as normalized fluorescence signal.

Colloidal stability of QD-LH and QD/QSY was evaluated in both physiological PBS buffer and cell culture medium at $37{ }^{\circ} \mathrm{C}$ for up to $120 \mathrm{~h}$; the fluorescence intensity was measured using the fluorospectrometer.

Cell Culture: Human embryonic kidney 293T cells were incubated in DMEM containing $10 \% \mathrm{FBS}$ and $1 \%$ antibiotics at $37^{\circ} \mathrm{C}$ in a humidified atmosphere and $5 \% \mathrm{CO}_{2}$.

In-Vitro Cytotoxicity Assay of the Prodrug-Type Probe: The cytotoxicity was studied in the non-tumoral cell line of 293T. Cells were treated with a series of concentrations of the prodrug-type probe in 96 -well plates with a standard MTT procedure. Absorbance was measured at $570 \mathrm{~nm}$ using a microplate reader (Thermo, USA). The cell viability was calculated according to the following equation, based on the optical densities of the sample, DMSO, and the control:

Cell viability $(\%)=$

$\left(O D_{\text {samples }}-O D_{\text {DMSO }}\right) /\left(O D_{\text {control }}-O D_{\text {DMSO }}\right) \times 100 \%$

Activation of the Prodrug-Type Probe using 293T-Leg Cell Lysates: Enzymatic activation was performed in the assay buffer $(40 \mathrm{~mm}$ citric acid, $121 \mathrm{~mm}$ disodium phosphate dodecahydrate, $1 \mathrm{~mm}$ EDTA, $0.1 \% 3$-[(3-cholamidopropyl) dimethylammonio] propanesulfonate (CHAPS), $1 \mathrm{~mm}$ dithioreitol (DTT), $\mathrm{pH} \mathrm{5.8),} \mathrm{and} \mathrm{the} \mathrm{prodrug-type} \mathrm{probe}$ was incubated with the $293 \mathrm{~T}$-Leg cell lysates or trypsin at $37{ }^{\circ} \mathrm{C}$ for $30 \mathrm{~min}$. Fluorescence intensity of the activated QD were measured at an excitation wavelength of $\lambda_{\mathrm{ex}}=260 \mathrm{~nm}$ and an emission wavelength of $\lambda_{\text {em }}=668 \mathrm{~nm}$.

Cellular Activation of the Prodrug-Type Probe: Cell-specific activation of the prodrug-type QD performed in the normal 293T cells and transfected 293T-Leg cells. The QD/QSY was incubated with cells for $5 \mathrm{~h}$. Cells were then washed with PBS three times and fixed with $4 \%$ paraformaldehyde. Extracellular fluorescence was quenched using trypan blue. Cells were observed using a fluorescence microscope (Olympus, Japan). Furthermore, the uptake efficiency was quantitatively measured by FACS. In brief, normal 293T cells and transfected 293T-Leg cells were exposed to the medium containing the prodrug-type probe for $5 \mathrm{~h}$. After washing with PBS, the cells were collected, and further washed with PBS three times. The cells were subjected to fluorescence analysis with FACS (BD, USA).

In-Vivo Animal Studies: Female BALB/c nude mice (4-5 weeks old, 18-22 g) were housed under specific pathogen-free conditions and in a $12 \mathrm{~h}$ light/dark cycle. Animals possessed free access to sterilized food pellets and distilled water. The experimental procedures were approved by the Institutional Animal Care and Use Committee.

Xenograft Tumor Mouse Models: Human colon cancer SW620 cells were incubated in RMPI 1640 medium containing 10\% FBS, and 1\% antibiotics at $37{ }^{\circ} \mathrm{C}$ under a humidified atmosphere and $5 \% \mathrm{CO}_{2}$. A xenograft tumor model was established by inoculating $1 \times 10^{6} \mathrm{SW} 620$ cells subcutaneously into the back of nude mice.

Tissue-Specific Activation: Mice were humanely killed and the major organs (heart, liver, spleen, lung, kidney, and tumor) collected for cryosectioning. The tissue slices were divided into two experimental groups: the non-treatment slices, and the enzyme-inactivated slices that were pretreated with $4 \%$ glutaraldehyde to denature legumain. The slides were exposed to the prodrug-type nanoprobe $(160 \mu \mathrm{g} / \mathrm{mL})$ for $30 \mathrm{~min}$ at room temperature, and then the activated fluorescence was imaged with a fluorescence microscope (Olympus, Japan).

In-Vivo Imaging: The mice bearing the SW620 tumor were given the prodrug-type QD via peritumoral or intramuscular injection for in-situ activation and imaging studies. Fluorescent imaging of the QD that was activated by legumain was obtained using the IVIS imaging system (Caliper Life Science) and analyzed using Living Image (Caliper Life Sciences). Furthermore, tail vein intravenous injection was conducted for investigating the application in real-time tumor imaging.

Statistical Analysis: The quantitative data were expressed as mean \pm standard deviation (S.D.). Statistical analysis was performed by the Student's $t$-test. Statistical significance was inferred at a value of $P<$ 0.05 . 

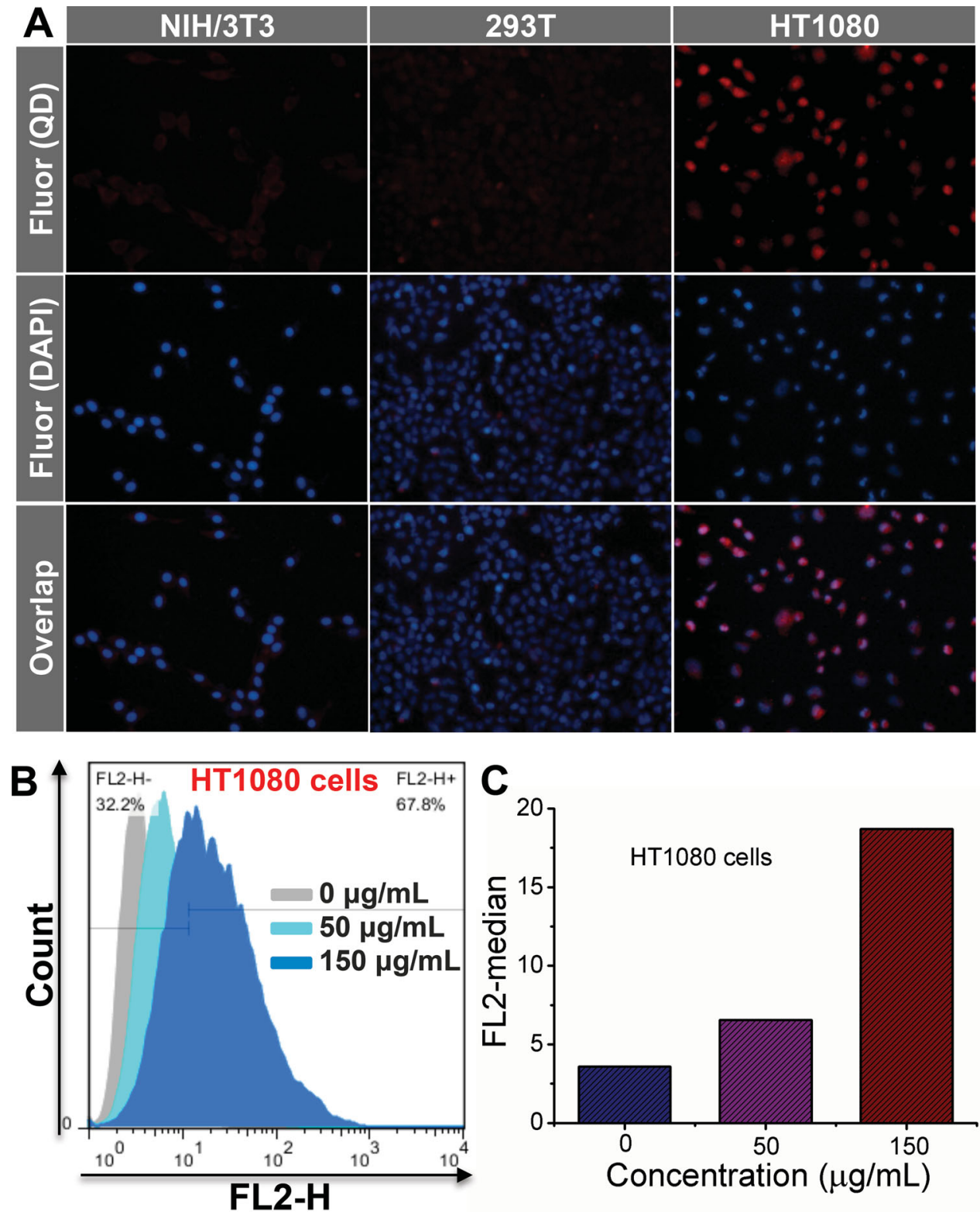

Figure 9. MMP-2-induced activation of the QD/QSY nanoprobe. A) Activation of the nanoprobe $(150 \mu \mathrm{g} / \mathrm{mL})$ in different cell lines, among which $\mathrm{NIH} / 3 \mathrm{~T} 3$ and HEK 293T cells lack MMP-2 expression, whereas HT1080 overexpresses MMP-2. Images presented are red fluorescence (QD), blue fluorescence (DAPI), and merged images. B) FACS assay results for MMP-2-induced activation efficiency in HT1080 at varying nanoprobe concentrations $(0,50$, and $150 \mu \mathrm{g} / \mathrm{mL})(\mathrm{FL} 2-\mathrm{H}$ : height of red fluorescence intensity). C) Median fluorescence intensity versus nanoprobe concentration in HT1080 cells.

\section{Acknowledgments}

This work was supported by the following grants: National Basic Research Program of China (973 Program 2014CB931900, 2013CB932503), and NSFC, China $(91029743,81172996,81373357,81361140344)$.

Received: February 7, 2014 Revised: May 4, 2014 Published online: July 7, 2014

[1] a) C. Lopez-Otin, J. S. Bond, J. Biol. Chem. 2008, 283, 30433; b) S. D. Mason, J. A. Joyce, Trends Cell. Biol. 2011, 21, 228.

[2] K. Y. Choi, M. Swierczewska, S. Lee, X. Chen, Theranostics 2012, 2, 156.
[3] G. Lee, K. Eom, J. Park, J. Yang, S. Haam, Y. M. Huh, J. K. Ryu, N. H. Kim, J. I. Yook, S. W. Lee, D. S. Yoon, T. Kwon, Angew. Chem. Int. Ed. Engl. 2012, 51, 5837.

[4] C. Liu, C. Sun, H. Huang, K. Janda, T. Edgington, Cancer Res. 2003, 63, 2957.

[5] a) N. Li, Q. Liu, Q. Su, C. Wei, B. Lan, J. Wang, G. Bao, F. Yan, Y. Yu, B. Peng, J. Qiu, X. Yan, S. Zhang, F. Guo, Med. Oncol. 2013, 30, 621; b) P. Guo, Z. Zhu, Z. Sun, Z. Wang, X. Zheng, H. Xu, PLoS One 2013, 8, e73090; c) L. Wang, S. Chen, M. Zhang, N. Li, Y. Chen, W. Su, Y. Liu, D. Lu, S. Li, Y. Yang, Z. Li, D. Stupack, P. Qu, H. Hu, R. Xiang, J. Cell Biochem. 2012, 113, 2679.

[6] a) Y. Luo, H. Zhou, J. Krueger, C. Kaplan, S. H. Lee, C. Dolman, D. Markowitz, W. Wu, C. Liu, R. A. Reisfeld, R. Xiang, J. Clin. 
Invest. 2006, 116, 2132; b) W. Wu, Y. Luo, C. Sun, Y. Liu, P. Kuo, J. Varga, R. Xiang, R. Reisfeld, K. D. Janda, T. S. Edgington, C. Liu, Cancer Res. 2006, 66, 970; c) D. Liao, Z. Liu, W. J. Wrasidlo, Y. Luo, G. Nguyen, T. Chen, R. Xiang, R. A. Reisfeld, Cancer Res. 2011, 71, 5688; d) J. J. Briggs, M. H. Haugen, H. T. Johansen, A. I. Riker, M. Abrahamson, O. Fodstad, G. M. Maelandsmo, R. Solberg, BMC Cancer 2010, 10, 17.

[7] M. A. Mathieu, M. Bogyo, C. R. Caffrey, Y. Choe, J. Lee, H. Chapman, M. Sajid, C. S. Craik, J. H. McKerrow, Mol. Biochem. Parasitol. 2002, 121, 99.

[8] L. Stern, R. Perry, P. Ofek, A. Many, D. Shabat, R. Satchi-Fainaro, Bioconjug. Chem. 2009, 20, 500.

[9] a) S. S. Naik, J. F. Liang, Y. J. Park, W. K. Lee, V. C. Yang, J. Control. Release 2005, 101, 35; b) Y. M. Kwon, Y. Li, S. Naik, J. F. Liang, Y. Huang, Y. J. Park, V. C. Yang, Expert Opin. Drug Delivery 2008, 5, 1255.
[10] J. Fang, H. Nakamura, H. Maeda, Adv. Drug Delivery Rev. 2011, 63, 136.

[11] Y. Huang, Y. S. Park, J. Wang, C. Moon, Y. M. Kwon, H. S. Chung, Y. J. Park, V. C. Yang, Curr. Pharm. Des. 2010, 16, 2369.

[12] X. Jiang, C. Rocker, M. Hafner, S. Brandholt, R. M. Dorlich, G. U. Nienhaus, ACS Nano 2010, 4, 6787.

[13] D. N. Li, S. P. Matthews, A. N. Antoniou, D. Mazzeo, C. Watts, J. Biol. Chem. 2003, 278, 38980.

[14] Y. Morita, H. Araki, T. Sugimoto, K. Takeuchi, T. Yamane, T. Maeda, Y. Yamamoto, K. Nishi, M. Asano, K. Shirahama-Noda, M. Nishimura, T. Uzu, I. Hara-Nishimura, D. Koya, A. Kashiwagi, I. Ohkubo, FEBS Lett. 2007, 581, 1417.

[15] T. Jiang, E. S. Olson, Q. T. Nguyen, M. Roy, P. A. Jennings, R. Y. Tsien, Proc. Natl. Acad. Sci. USA 2004, 101, 17867. 